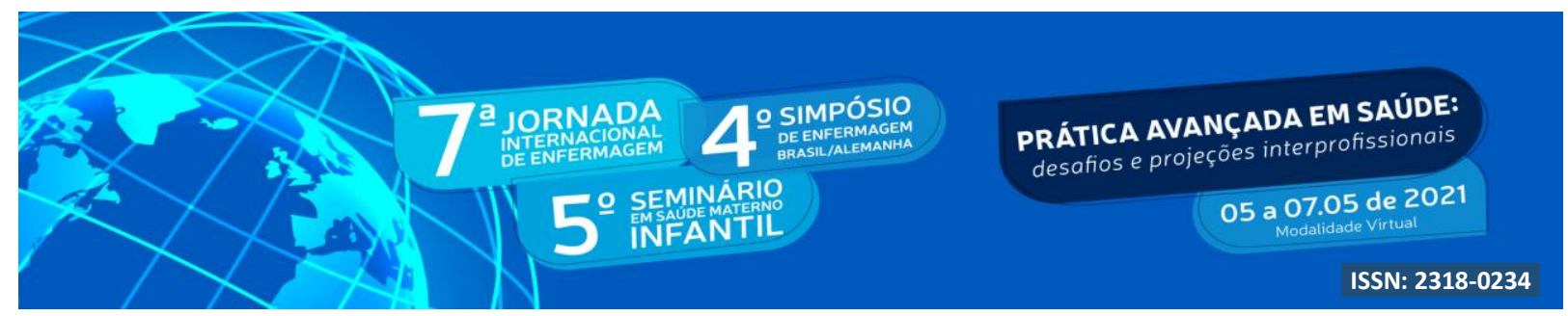

DOI: http://doi.org/10.48195/jie2021-046

\title{
TECNOLOGIAS EDUCACIONAIS VALIDADAS PARA PESSOAS COM HIPERTENSÃO ARTERIAL SISTÊMICA: ESTUDO DE TENDÊNCIAS ${ }^{1}$
}

\author{
Dedabrio Marques Gama²; Laís Mara Caetano da Silva ${ }^{3}$; Maria Denise Schimith ${ }^{4}$; \\ Marcelo Nunes da Silva Fernandes ${ }^{5}$; Adriany da Rocha Pimentão ${ }^{6}$, Daiana Cristina \\ Wickert $^{7}$
}

\begin{abstract}
RESUMO
Objetivou-se conhecer as tendências da produção científica em dissertações e teses relacionadas as tecnologias educacionais validadas para pessoas com hipertensão arterial sistêmica. Trata-se de uma revisão bibliográfica realizada em dezembro de 2020 no Catálogo de Teses e Dissertações da Coordenação de Aperfeiçoamento de Pessoal de Nível Superior, totalizando seis estudos. Cinco estudos eram da área da enfermagem e um da saúde coletiva. Um estudo foi publicado em 2014, dois em 2015, dois em 2016 e um em 2017. Dois estudos eram da Universidade Estadual do Ceará, um da Universidade de Fortaleza, um da Universidade Federal de Alfenas e dois do Centro Universitário São Camilo. Três estudos eram da região sudeste e três do nordeste. Quanto ao tipo de estudo, um se tratava de pesquisa de abordagem avaliativa, dois de estudos metodológicos, dois ensaios clínicos randomizados e um estudo do tipo multi-método. O estudo possibilitou evidenciar que existem poucas produções na temática, contribuindo com a identificação da relevância da pesquisa.
\end{abstract}

Palavras-chave: Enfermagem; Tecnologia educacional; Hipertensão; Educação em saúde.

\begin{abstract}
The objective was to know the trends of scientific production in dissertations and theses related to validated educational technologies for people with systemic arterial hypertension. This is a bibliographic review carried out in December 2020 in the Catalog of Theses and Dissertations of the Coordination for the Improvement of Higher Education Personnel, totaling six studies. Five studies were in the field of nursing and one in collective health. One study was published in 2014, two in 2015, two in 2016 and one in 2017. Two studies were from the State University of Ceará, one from the University of Fortaleza, one from the Federal University of Alfenas and two from the São Camilo University Center. Three studies were from the southeastern region and three from the northeast. As for the type of study, one was a research with an evaluative approach, two were methodological

\footnotetext{
${ }^{1}$ Estudo de tendências.

${ }^{2}$ Enfermeiro. Mestrando em Enfermagem - PPGEnf/UFSM. dedabrio.gama@gmail.com

${ }^{3}$ Enfermeira. Orientadora. Professora do Curso de Enfermagem - UFSM. lais.silva@ufsm.br

${ }^{4}$ Enfermeira. Coorientadora. Professora do Curso de Enfermagem - UFSM. ma.denise2011@gmail.com

${ }^{5}$ Enfermeiro. Doutorando em Enfermagem - PPGEnf/UFRGS. marcelonsf@gmail.com

${ }^{6}$ Enfermeira. Mestranda em enfermagem - PPGEnf/UFSM. adry.pimentao@ gmail.com

${ }^{7}$ Enfermeira. Mestranda em enfermagem - PPGEnf/UFSM. daianacristinaw@ gmail.com
} 


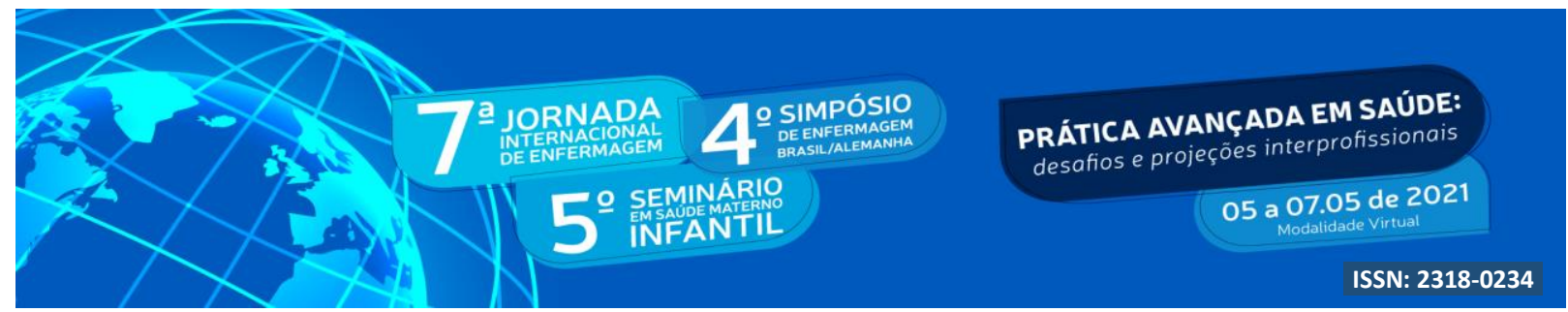

studies, two randomized clinical trials and a multi-method study. The study made it possible to show that there are few productions on the theme, contributing to the identification of the relevance of the research.

Keywords: Nursing; Educational technology; Hypertension; Health Education.

\section{INTRODUÇÃO}

As doenças crônicas não transmissíveis (DCNT) são um conjunto de doenças que podem levar à incapacidade e morte precoce, tornando-se um dos maiores problemas de saúde pública no Brasil (TERZIC; WALDMAN, 2011; SILVA; LUIZ; PEREIRA, 2015). Dentre essas doenças destaca-se a Hipertensão Arterial Sistêmica (HAS). A HAS é uma doença cardiovascular com manifestações clínicas de origem multifatorial e um dos principais fatores de risco cardiovasculare (BRASIL, 2013).

No Brasil, a HAS está entre as DCNT com maiores índices de morbimortalidade, atingindo 32,5\% dos adultos (SOCIEDADE BRASILEIRA DE CARDIOLOGIA, 2016). Presume-se que até 2025 os casos de HAS aumentem em 60\% em todo o mundo (MALTA et al., 2017).

Dessa forma, torna-se fundamental o controle desta doença, pois seu manejo inadequado compromete a qualidade de vida dos indivíduos. Uma das estratégias utilizadas é a educação em saúde. Trata-se de uma ferramenta fundamental que busca articular saberes científicos e populares para atuar sobre uma determinada situação, de modo que a pessoa se perceba como o agente de transformação de sua própria vida (SOUZA et al., 2010).

A educação em saúde pode ser desenvolvida pelo uso de tecnologias educacionais, visando contribuir com atividades de ensino e de aprendizagem (ASSUNÇÃO et al., 2013). No que se refere a validação de tecnologias educacionais, é necessário envolver a comunidade no intuito de que as investigações possam gerar novas tecnologias, considerando o propósito do pesquisador e a ferramenta que se pretende validar (TEIXEIRA, 2010).

Com a crescente validação de tecnologias, o estudo justifica-se pela possibilidade de ofertar uma ferramenta que favoreça a autonomia e tomada de decisão das pessoas com HAS por meio de iniciativas que ampliem os conhecimentos relacionados aos recursos produzidos 


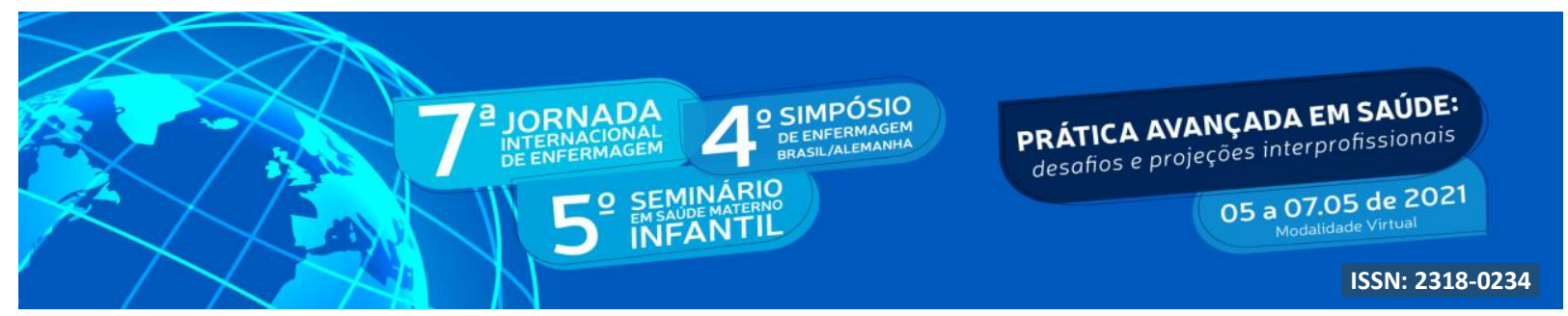

para a educação em saúde. Assim, a fim de aprofundar conhecimentos sobre os estudos de validação, buscou-se realizar um estudo bibliográfico, buscando responder ao seguinte questionamento: Qual a tendência da produção científica em dissertações e teses relacionada as tecnologias educacionais validadas para as pessoas com hipertensão arterial sistêmica? E, como objetivo, tem-se: conhecer as tendências da produção científica em dissertações e teses relacionadas as tecnologias educacionais validadas para pessoas com hipertensão arterial sistêmica.

\section{OBJETIVO}

Conhecer as tendências da produção científica em dissertações e teses relacionadas as tecnologias educacionais validadas para pessoas com hipertensão arterial sistêmica.

\section{MÉTODO}

Trata-se de uma revisão bibliográfica da literatura desenvolvida na disciplina “Construção do Conhecimento em Saúde e Enfermagem”, vinculada ao Programa de Pósgraduação em Enfermagem da Universidade Federal de Santa Maria (UFSM). Para atingir o objetivo proposto, a coleta dos dados foi realizada no mês de julho de 2020 , sendo utilizado o Catálogo de Teses e Dissertações da Coordenação de Aperfeiçoamento de Pessoal de Nível Superior (CAPES).

A estratégia de busca para o levantamento das produções científicas foi tecnologia educacional AND hipertens*. As dissertações e teses foram selecionadas de acordo com os seguintes critérios de inclusão: dissertações e teses com resumos completos e relacionados com a temática. Foram excluídos os estudos com resumos incompletos e que não se relacionassem com a temática. Não foi utilizado recorte temporal.

Assim, ao empregar as palavras, obtiveram-se 85 estudos, sendo 22 teses e 63 dissertações. Das dissertações, 14 eram de mestrado profissional e 49 de mestrado acadêmico. Seguindo aos critérios de inclusão e exclusão, fizeram parte da amostra seis estudos, sendo duas teses e quatro dissertações, das quais duas eram de mestrado profissional e duas de 


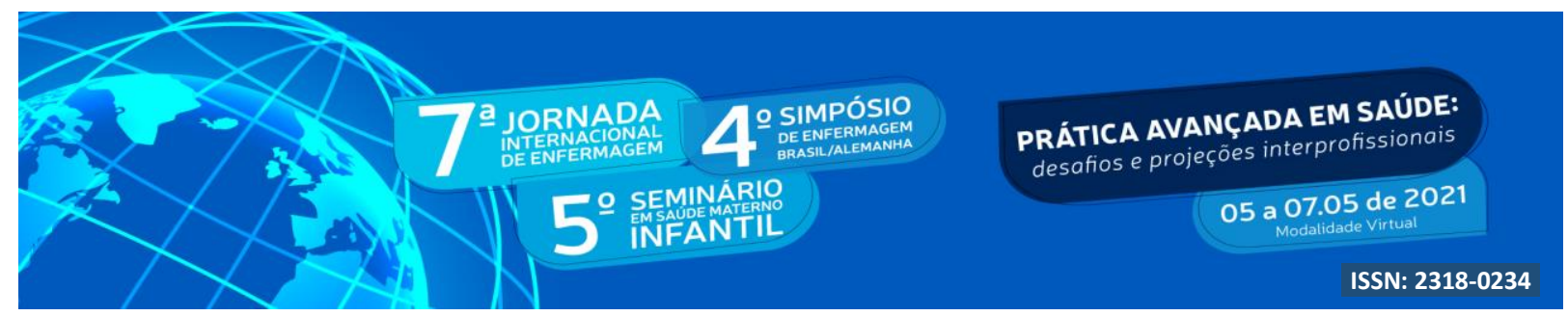

mestrado acadêmico. A análise e síntese dos dados foram realizadas após a leitura exaustiva dos resumos das dissertações e teses.

\section{RESULTADOS E DISCUSSÃO}

Dentre as dissertações e teses, cinco eram da área de conhecimento da enfermagem e da área de Saúde Coletiva. Quanto ao ano de publicação, um estudo foi publicado em 2014, dois em 2015, dois em 2016 e um em 2017. A área da enfermagem destacou-se na produção dos estudos, buscando a promoção da saúde, a prevenção de agravos e complicações e a corresponsabilização do usuário com o seu cuidado (MENDES; SILVA; FERREIRA, 2018).

Entre os estudos selecionados, dois foram desenvolvidos na Universidade Estadual do Ceará, um na Universidade de Fortaleza, um na Universidade Federal de Alfenas, e dois no Centro Universitário São Camilo. Três estudos eram da região sudeste e três estudos eram da região nordeste. Foi realizada uma pesquisa de abordagem avaliativa por meio da triangulação de métodos, dois estudos metodológicos, dois ensaios clínicos randomizados e um estudo do tipo multi-método.

Os objetivos dos estudos foram testar a eficácia de uma tecnologia educacional (álbum seriado) como subsídio ao enfermeiro para promoção da qualidade de vida e adesão ao tratamento em pessoas com hipertensão arterial; descrever o processo de construção e validação de uma tecnologia educativa para pessoas com doença renal crônica; avaliar as estratégias de ensino e de aprendizagem para a formação interdisciplinar e integral em saúde com o apoio de tecnologias; avaliar a influência do uso da tecnologia educacional na modalidade híbrida blended learning (ensino presencial associado por meio do ambiente virtual de aprendizado "E-Care da Hipertensão") com vistas a melhoria do controle da pressão arterial; testar a eficácia de uma cartilha educativa sobre excesso ponderal no favorecimento do estágio de prontidão para mudança dos hábitos alimentares e atividade física, ao utilizá-la como subsídio para a prática do enfermeiro na educação em saúde do adulto com hipertensão; avaliar a influência da tecnologia educacional na modalidade do ensino a distância (EaD) no Ambiente Virtual De Aprendizagem (AVA) "E-care da hipertensão" sobre a adesão terapêutica em pessoas hipertensas que possuem difícil controle dos níveis pressóricos. 


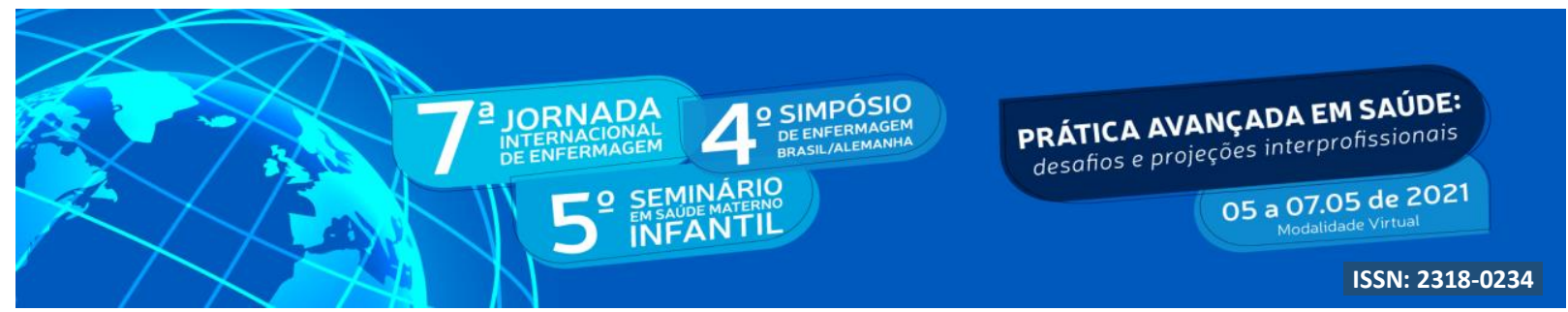

As tecnologias das produções analisadas possibilitaram informar os usuários sobre a HAS, buscando contribuir com uma melhor qualidade de vida. Portanto, as tecnologias podem ser utilizadas, dentre outras funções, para a promoção do cuidado (PINTO et al., 2017). Assim, as tecnologias voltadas para a HAS favorecem o controle da condição e, consequentemente, contribuem com a diminuição da morbimortalidade por desfechos cardiovasculares.

\section{CONCLUSÃO}

Este estudo teve como objetivo conhecer as tendências da produção científica em dissertações e teses relacionadas as tecnologias educacionais validadas para pessoas com HAS. Considera-se que as tecnologias analisadas são importantes para a prevenção e controle da HAS, possibilitando a autonomia dos usuários. O estudo possibilitou evidenciar que existem poucas acerca da temática, e uma ausência de processo de validação no contexto gaúcho, o que contribuiu com a identificação da relevância da pesquisa e reforçou a necessidade de ampliação do conhecimento nessa temática.

\section{REFERÊNCIAS}

ASSUNÇÃO, A.P.F DE; BARBOSA, C.R.; MEDEIROS, H.P.; TAVARES, I.C.; SABÓIA, V.M. Práticas e tecnologias educacionais no cotidiano de enfermeiras da estratégia saúde da família. Rev. Enferm. UFPE. v.7, n.11, p. 6329-6335, 2013.

BRASIL. Ministério da Saúde. Secretaria de Atenção à Saúde. Departamento de Atenção Básica. Estratégias para o cuidado da pessoa com doença crônica: hipertensão arterial sistêmica. Brasília: Ministério da Saúde, 2013, 128 p. Disponível em: \&lt;http://dab.saude.gov.br/portaldab/biblioteca.php?conteudo=publicacoes/cab37\&gt; Acesso em 10 mar. 2021.

MALTA, D.C. BERNAL, R.T.I.; ANDRADE, S.S.C.A; SILVA, M.M.A.; VELASQUEZMELENDE, G. Prevalência e fatores associados com hipertensão arterial autorreferida em adultos brasileiros. Rev. Saúde Pública, São Paulo. v.51, n.1, jun. 2017. Disponível em: \&lt;http://dx.doi.org/10.1590/s1518-8787.2017051000006\&gt; Acesso em 10 mar. 2021.

MENDES, F.A.; SILVA, M.P.; FERREIRA, C.R.S. Diagnósticos de enfermagem em portadores de hipertensão arterial na atenção primária. Estação Científica (UNIFAP), v.8, 


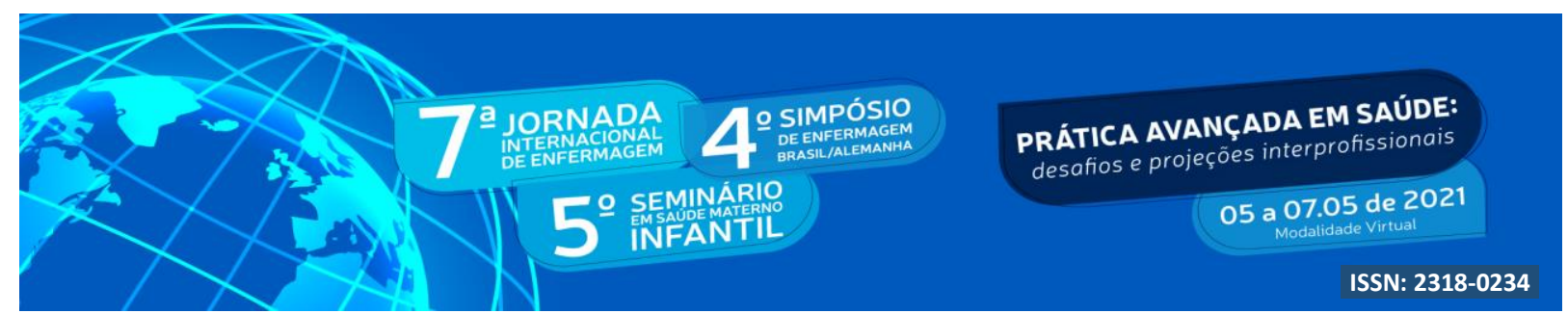

n.1, p.91-101., 2018.

PINTO, A.C.; SCOPACASA, L.G.; BEZERRA, L.L.A.L.; PEDROSA, J.V.; PINHEIRO, P.N.C. Use of information and communication technologies in health education for adolescents: integrative review. Revista de Enfermagem UFPE on line, v.11, n.2, p.634644, 2017.

SILVA, S.M.; LUIZ, R.R.; PEREIRA, R.A. Fatores de risco e proteção para doenças cardiovasculares em adultos de Cuiabá, Mato Grosso, Brasil. Rev. Bras. Epidemiol. v.18, n.2, p.425-438, 2015.

SOCIEDADE BRASILEIRA DE CARDIOLOGIA. $7^{a}$ Diretriz Brasileira de Hipertensão Arterial. Arq Bras Cardiol, Rio de Janeiro, v.107, n.3, set. 2016. Disponível em: \&lt;http://publicacoes.cardiol.br/2014/diretrizes/2016/05_HIPERTENSAO_ARTERIAL.pdf \&gt; Acesso em 10 mar. 2021.

SOUSA, L.B.; TORRES, C.A.; PINHEIRO, P.N.C.; PINHEIRO, A.K.B. Práticas de educação em saúde no Brasil: a atuação da enfermagem. REUERJ. v.18, n.1, p.55-60, 2010.

TEIXEIRA, E. Tecnologias em enfermagem: produções e tendências para a educação em saúde com a comunidade. Rev. Eletr. Enferm. v.12, n.4, 598 p. Disponível em: http://www.fen.ufg.br/revista/v12/n4/v12n4a01.htm. Acssso em 10 mar. de 2021.

TERZIC, A.; WALDMAN, S. Chronic diseases: the emerging pandemic. Clinical and translational science. v.4, n.3, p. 225-226, 2011. 